\title{
Ferdinand de Saussure y el Curso de lingüística general ${ }^{1}$
}

Xavier Laborda

Universidad de Barcelona (España)

xlaborda@ub.edu

\section{Resumen}

¿Cuál es la vigencia del paradigma saussureano? Como respuesta a esta pregunta se presenta en este breve escrito dos tesis en apariencia contradictorias. La primera es que no hay tal paradigma saussureano; negamos su existencia. Y la segunda es que, aun así, el paradigma saussureano tiene un peso destacable en la lingüística actual; afirmamos su vigencia relativa.

Palabras clave: paradigma kuhneano, Saussure, CLG, historia de la lingüística

\section{Ferdinand de Saussure and the Course of general linguistics}

\begin{abstract}
What is the validity of the Saussurean paradigm? In response to this question, two apparently contradictory theses are presented in this short paper. The first is that there is no Saussurean paradigm; We deny its existence. And the second is that, even so, the Saussurean paradigm has a remarkable weight in today's linguistic; We affirm its relative validity.
\end{abstract}

Keywords: Kuhnian paradigm, Saussure, CLG, history of linguistics

1 Artículo publicado en la revista Tonos Digital, número 4, diciembre de 2002. https://digitum.um.es/xmlui/ bitstream/10201/50967/1/Ferdinand\%20de\%20Saussure.pdf 
Ahí está la figura del celebrado Ferdinand de Saussure, de la cual hablamos a propósito del centenario de la publicación de su Curso de lingüistica general (1916). Nuestro comentario es breve y responde a la pregunta sobre la vigencia del paradigma saussureano en la lingüística actual. Vaya por delante que nuestra opinión se resume en dos ideas, en apariencia contradictorias. La primera es que no hay tal paradigma saussureano; negamos su existencia. Y la segunda es que, aun así, el paradigma saussureano tiene un peso destacable en la lingüística actual; afirmamos su vigencia relativa.

\section{A la sombra del fundador}

Uno ha de preguntarse si en esa cuestión tan interesante de su vigencia no se vinculan, inadvertidamente quizá, varios elementos. El más evidente es el del modelo estructuralista, que tiene el mérito superlativo de establecer la lingüística moderna, si no es el caso que sea simplemente la lingüística la que con Saussure se inaugura. En un estudio sucinto y perspicaz, John E. Joseph (1) repasa los principios del modelo saussureano, a saber, la primacía del lenguaje hablado, la lengua como objetivo de la ciencia, la semiología y la lengua como sistema de signos, la arbitrariedad del signo lingüístico, la linealidad de los significantes, las relaciones sintagmáticas y paradigmáticas, la lengua como forma y sistema.

Con esta aportación, que ha abastecido teoréticamente la lingüística de dos tercios del siglo XX, basta para explicar la atención, si no es admiración, que despierta el Curso de lingüística general. Como tantas veces se ha recordado, la anécdota es que la obra fue publicada en 1916 por iniciativa de compañeros y alumnos de Saussure, porque éste había fallecido en 1913 sin haber dado a la imprenta sus renovadoras ideas. Sin embargo, la anécdota trasciende sus circunstancias y se convierte en un elemento significativo.

Y en este plano de la biografía intelectual y de los avatares de la divulgación de su obra hallamos un segundo componente, menos llamativo pero recurrente en la historia de la lingüística. No deja de ser sorprendente y aleccionadora esta cadena de factores propiciatorios de la edición del Curso. Las reimpresiones y ediciones con ligeros retoques de compaginación son una cara de la fortuna de la obra. La edición de las fuentes manuscritas por Robert Godel, en 1957, y de otros documentos complementarios entre 1957 y 1959, expresa el carácter serial de esta obra, que por razón de ser un paradigma de las ciencias provoca ese interés de descubrir los estratos documentales del modelo. Nuevas ediciones, como la de T. Mauro y con epílogo de Louis Calvet, en 1985, o la reciente de 1993 con las notas tomadas por el alumno Émile Constantin en el último curso (1910-1911), desmienten la suposición de que todas las cartas estén sobre la mesa.

Este segundo plano, el de la biografía intelectual y el tránsito de sus ideas, tiene un claro componente mítico, que despierta una contenida $\mathrm{y}$ discreta fascinación por el fundador y su esquivo rastro. Es tradicional atribuir el origen cierto de cada una de las ciencias, especialmente las que tienen una historia antigua, a un fundador. La figura de una autoridad individual infunde una gran reverencia $y$, por otra parte, señala con claridad un origen concreto, tan concreto y deslumbrante como el de esa personalidad. Las leyendas fundacionales son comunes y dan lustre. Es el caso de la retórica, con la figura de Córax, gran maestro que según la tradición debe litigar con su discípulo Tísias. La gramática dispone de su fundador con Dionisio el Tracio y la Téchne grammatiké a él atribuida, aunque no sin polémicas. $\mathrm{Y}$ en la lingüística se reproduce también ese apreciado fenómeno narrativo de proyectar sobre ella el esplendor de una figura.

A su modo, Saussure cumple en el imaginario de los lingüistas ese papel de fundador aureolado de episodios paradójicos: el alejamiento sin estridencias de su formación como neogramático, un silencio sobre sus nuevas formulaciones propio de un ideal budista, la intervención providencial de discípulos y compañeros en la edición póstuma, el empuje difusor de su obra por la francofonía suiza, la dilatada tarea de las ediciones críticas, la atribución errónea de ideas y la polémica (como la suscitada por 
Jakobson) contra éstas. Por mencionar sólo dos aspectos biográficos, en primer lugar, llama la atención que Saussure no publicara apenas nada desde 1897, fecha en que apareció su trabajo sobre gramática comparada, Mémoire. Esta obra también fue destacable en su momento y mereció una cálida acogida en París, donde Saussure impartió la docencia entre 1881 y 1891 y participó activamente de la sociedad científica. Las investigaciones historiográficas de K. Koerner sobre Saussure revelan la complejidad cultural de su obra; y éste es un segundo punto de la cuestión. Como ha señalado Koerner, la formulación saussureana surge de un "estado de opinión contemporáneo", de un ambiente científico, que pertenece a su época pero que sólo él es capaz de desarrollar en un nuevo paradigma de lingüística axiomática. El estudio de estas fuentes, fragmentarias y contradictorias, que le animaron a considerar la comunicación a partir de la teoría del signo y del valor de sus elementos de las lengua por contraste, es un trabajo que remonta y desdice esa visión legendaria y simplista del fundador.

\section{Estructuralismo y paradigma de ciencias}

También es cierto que importa mucho más la tradición que instaura el Curso de lingüística general que cualquier consideración sobre sus causas científicas o la reconstrucción de la documentación canónica de su pensamiento. El desarrollo de las disciplinas de la lingüística, a partir de su paradigma, es impresionante: fonología, sintaxis, estilística, análisis textual, etc. En lo que se refiere a la tradición, si tomamos como referencia a un lingüista llamado a ocupar un lugar en esta misma sección [...], Noam Chomsky, apreciamos que éste ha tenido muy presente la obra de Saussure y que se ha referido a él con frecuencia, especialmente entre 1962 y 1972 (2).

Si acudimos a los historiadores, es decir, a los narradores del devenir de la lingüística, podemos obtener calas sobre el juicio que hacen de la obra de Saussure. Este procedimiento es elemental, pero revelador. Tomamos como referencia los manuales de historiadores tan reputados como R. H. Robins (1967), Jesús Tusón (1982) y Bertil
Malmberg (1991). En la obra de R. H. Robins (Breve historia de la lingüística, 1967), Saussure aparece más veces que Chomsky, pero muchas menos que Prisciano y Bloomfield; Saussure es el quinto autor más nombrado. En la obra de Jesús Tusón (Aproximación a la Historia de la Lingüística, 1982), Saussure ocupa el tercer lugar en referencias, después de Chomsky y PortRoyal. Por su parte, B. Malmberg (Histoire de la linguistique. De Sumer à Saussure, 1991), a pesar de que sólo considera el Saussure comparatista, coloca a éste en la cabeza de sus referencias, seguido muy de cerca por Humboldt, Grimm y muchos otros comparatistas.

Habrá de verse en estas preferencias de los historiadores no tan sólo los rasgos de sus autores, sino también el influjo de una perspectiva científica o paradigma que les orienta en la interpretación del pasado. Robins se muestra conciliador del presente con la tradición grecolatina, $\mathrm{y}$ ofrece este orden referencial de autores: Bloomfield, Prisciano, estoicos, Dionisio de Tracia y Saussure. Tusón combina los dos paradigmas fundamentales del siglo $\mathrm{XX}$, estructuralismo y generativismo, tal como atestigua el rastro de sus referencias: Chomsky, Port-Royal, Saussure, Prisciano y los Modistae. Malmberg, a su vez, se muestra particularmente atraído por los autores adscritos al paradigma comparatista.

Acudimos a otro historiador, K. Koerner (3), quien ha establecido que en los dos últimos siglos han reinado tres paradigmas. Toma su denominación de los nombres de sus fundadores (nuevo impulso a las leyendas fundacionales). El del s. XIX es el schleicheriano o comparatista, que considera las lenguas como materia de tipologización y como productos históricos (4). $\mathrm{Y}$ los paradigmas del s. XX son el saussureano o estructuralista y el chomskiano o generativista que, en la parte que tienen en común, se interesan por las relaciones, oposiciones, categorías y normas que subyacen en el sistema del lenguaje. Propio del estructuralismo saussureano es tomar las lenguas indistintamente como medios de cognición y de expresión de la condición social e individual del sujeto. 
Como se ha visto, los paradigmas comparatista, estructuralista y generativista resultan determinantes a la hora de narrar una historia de la lingüística selectiva y coherente. Pero no sólo muestran su protagonismo en la narración o, lo que es lo mismo, no sólo hablan del pasado, sino que también se refieren a un presente productivo. ¿Qué significa ello? Significa que los historiadores, que tienen por objeto hablar del pasado en clave de actualidad, al escoger una particular perspectiva, enseñan que los principios de su paradigma siguen vigentes. Así lo declara Ranko Bugarski (5) cuando describe las cuatro corrientes de la lingüística del s. XIX y del s. XX. Primera, la filológica de carácter diacrónico o comparatista. Segunda, la socio-psicológica, con Humboldt, Sapir y Saussure, entre sus cultivadores, y que se centra en aspectos de la cognición y la expresión tanto social como individual de la persona. Tercera, la descriptiva, afecta al estudio de la conducta verbal y, en particular, de las lenguas sin tradición escrita, tal como la perfiló Bloomfield. Y una cuarta corriente, que reúne a Saussure y Chomsky, Trubetzkoy y Hjelmslev, de carácter axiomático, dedicada a establecer los principios de la cognición y del lenguaje.

No se podría pedir mayor claridad a las palabras de Bugarski. En primer lugar, establece que la lingüística progresa por el empuje de corrientes de investigación, delimitadas mediante una concepción distintiva de su objeto de estudio y de los principios metodológicos. En segundo lugar, reconoce que esas corrientes desbordan en algunos casos los límites de los paradigmas, de modo que hay zonas de contacto e intercambio entre tales paradigmas. Esta visión compleja de la historia tiene el mérito de trascender la cronología y de agrupar en torno a una concepción de la realidad lingüística autores de épocas diferentes. Y, en tercer y último lugar, afirma que esas cuatro corrientes siguen siendo válidas y que están frecuentadas por investigadores que perseveran en sus particulares concepciones. De estos comentarios de Bugarski retenemos dos aspectos. Por una parte, nos parece notablemente perspicaz su aseveración sobre la ambigüedad de la idea de paradigma, puesto que, como señala, hay conexiones que históricamente superan y enriquecen esas formulaciones teóricas. $Y$, por la otra, conviene recordar la relativa actualidad de todas estas líneas de investigación -comparada, estructural y generativa-, ocupadas en tareas tan atractivas como la tipología de las lenguas, los mecanismos de semiosis o los patrones de rección sintáctica, respectivamente. De ahí que, como anunciábamos, nuestra opinión se resuma en que no hay propiamente un paradigma saussureano sino una gran corriente, y es así que sus aspectos tienen un peso destacable en la lingüística actual.

Volviendo al paradigma saussureano, ya para terminar, podemos recordar el comentario de Koerner (6) sobre cuáles son los ingredientes que el maestro ginebrino tomó de su época y de qué asombroso modo los articuló. "Su contribución está - expone Koerner - en que absorbió ideas sobre la naturaleza social del lenguaje, sobre el valor relativo de sus elementos y sobre su mecanismo de significación organizado de modo sistemático, ideas estas conocidas a fin de siglo, pero reunidas en un sistema coherente e independiente de cualquier fuente." Pierre Swiggers consigue expresar de un modo rotundo el valor de su obra: "el gesto de Ferdinand de Saussure será mostrar a los lingüistas qué es lo que hacen cuando practican su disciplina" (7). $Y$ esto fue una verdadera novedad en su época. Saussure fue una personalidad única. No sólo practicó la lingüística sino que también teorizó sobre ella. Y, además, se abstuvo de publicar al respecto (8).

Es imperdonable que a estas bajuras de nuestro escrito no hayamos mencionado aún un mérito universal del paradigma saussureano $y$ del estructuralismo. En el siglo $X X$, un siglo que científicamente ha girado sobre los problemas del lenguaje, y pensamos en ciencias como la filosofía, la matemática, la historia, la antropología, la arquitectura, por ejemplo, en ese siglo - como decíamos - el estructuralismo ha sido precisamente el paradigma de las ciencias, que ya es desempeñar un papel deslumbrante. Pero he aquí que, encabalgado sobre las consideraciones historiográficas, un pensamiento irreverente nos interfiere el discurso, se impone y exige salir a la 
luz. Se trata del diálogo de la duquesa de Alicia en el país de las maravillas, que se exclama así: "Si la gente no metiera las narices en lo que no les importa, el mundo giraría más deprisa". Es decir, traduciéndolo a nuestro propio mundo, que si los lingüistas no se anduvieran por las ramas de la historia, la ciencia avanzaría más. La crítica de la duquesa no por absurda es menos frecuente en el mundo académico, generalmente dirigida contra los quebraderos de cabeza historiográficos y esa obcecación por teorizar sobre el proceso histórico de la lingüística, como si no tuviéramos bastante con practicar la lingüística. Alguien verá en esta polémica un inexplicable y desdichado desaprovechamiento de la principal enseñanza de Saussure: no basta con hacer, sino que también se debe pensar sobre qué y cómo se hace.

\section{References}

1. J. E. Joseph, "Saussurean Tradition in Linguistics", in K. Koerner, R. Asher, Concise History of the Language Sciences, Oxford, Pergamon, 1995.
2. Véase K. Koerner, Professing Lingüistic Historiography, Amsterdam, John Benjamins, 1995, pág. 102.

3. K. Koerner, "Towards Historiography of Linguistics", in H. Parret (ed.): History of Linguistic Thought and Contemporary Linguistics, Berlin-New York, Walter de Gruyter, 1976, pág. 685s.

4. Pierre Swiggers, Histoire de la pensée linguistique, Paris, Puf, 1997, pág. 207s.

5. R. Bugarski, "The Object of Linguistics in Historical Perspective", in H. Parret (ed.): History of Linguistic Thought and Contemporary Linguistics, Berlin-New York, Walter de Gruyter, 1976, pág. 3.

6. K. Koerner, "Towards Historiography of Linguistics”, ídem, 1976, pág. 703.

7. P. Swiggers, Histoire de la pensée linguistique, Paris, Puf, 1997, pág. 262.

8. Véase P. Swiggers, Languages and Linguists: Aims, perspectives and duties of linguistics, Leuven-Paris, Peeters, 1997, pág. 55. 